Wien klin Mag 2021 · 24:34-42 https://doi.org/10.1007/s00740-020-00370-0 Online publiziert: 23. November 2020 (c) Der/die Autor(en) 2020

Lena Hieggelke - Anne M. Schultheis

Institut für Pathologie, Uniklinik Köln, Köln, Deutschland

\title{
Anwendungen der FISH in der Diagnostik von Lungenkarzinomen
}

Untersuchung von $E G F R, B R A F, A L K$, ROS1 sowie auch der PD-L1-Expressionsstatus gefordert $[16,20]$. Idealerweise sollten die molekularen Analysen auf die neueren Zielstrukturen MET, RET, ERBB2, NTRK und KRAS ausgeweitet werden ([20]; • Abb. 1). Bislang wird für LUSC nur die PD-L1-Testung verlangt [20], aber auch hier entwickelt sich das Feld der personalisierten Therapie weiter (•Abb. 1.) Die Fluoreszenz-in-situHybridisierung (FISH) hat früh Eingang in die Routinediagnostik des NSCLC gefunden, da sie es erlaubt, rasch, präzise und gewebesparend bestimmte genetische Veränderungen nachzuweisen. Obwohl neuere, umfassendere molekulare Methoden, wie z.B. die (RNA-)sequenzierungsbasierte Fusionsdetektion, an Aufmerksamkeit gewinnen, ist die FISH nach wie vor der Goldstandard zum Nachweis von therapierelevanten Gentranslokationen und Amplifikation im NSCLC.

Besondere Schwierigkeiten können sehr kleine Gewebeproben, artifiziell alteriertes Gewebe, Nekrose- oder Entzündungsareale mit vielen Makrophagen (falsch positive Sondenanreicherung) oder Fixierungsartefakte hervorrufen. Daher sollten alle Fälle immer (zusätzlich) von einem erfahrenen Auswerter gesichtet werden, der die zahlreichen möglichen Fallstricke kennt und interpretieren kann.

Ein aussagekräftiger Befund, der neben dem Ergebnis auch die verwendetet Sonde, die Anzahl der gezählten Tumorzellen sowie den Schwellenwert der verwendeten Sonde angibt und vor allem das Untersuchungsergebnis in den kli- nischen Kontext einordnet, ist bei jeder Analyse wünschenswert.

\section{ALK}

Eine $A L K$-Translokation in Form einer inversen Fusion mit dem EML4-Gen wurde erstmals 2007 nachgewiesen [24]. Mittlerweile sind über 15 EML4-ALKFusionsvarianten und weitere Fusionspartner bekannt [26]. Für die 3-5\% der Patienten mit ALK-positivem NSCLC ist seit 2012 Crizotinib (Xalkori ${ }^{\circledR}$, Pfizer Oncology, New York, USA) durch die European Medicines Agency (EMA) zugelassen ohne Kopplung an eine festgelegte Nachweismethode [1]. Gängige $A L K$ Dual-Colour-Break-Apart-Sonden markieren das $3^{\prime}$ - (rot) und das $5^{\prime}$ - (grün) Ende von ALK. Sind $5^{\prime}$ und $3^{\prime}$-Signale einer Tumorzelle verschmolzen, liegt definitionsgemäß ein Fusionsmuster vor ( $A L K$-negativ). Ein Bruchmuster ist definiert durch separate $5^{\prime}$ - und $3^{\prime}$-Signale in einem Abstand von 2 oder mehr Signaldurchmessern in einer Tumorzelle (•Tab. 1). Dieser Abstand wurde basierend auf Testungen mit dem Vysis LSI ALK Break-Apart FISH Probe Kit (Abbott Laboratories, Illinois, USA.) festgelegt. Wird ein anderes analytisches Reagenz verwendet, kann die Sondengröße variieren, was Unterschiede in der Definition eines Bruchmusters zur Folge haben kann [26]. Generell empfiehlt es sich, immer die Anweisungen des jeweiligen Herstellers zu befolgen. Bei einer ALK-EML4Inversion kann der Abstand auch kleiner sein (die Gene liegen in unmittelbarer Nachbarschaft voneinander auf Chromosom 2). Eine EML4/ALK-Tricheck- 


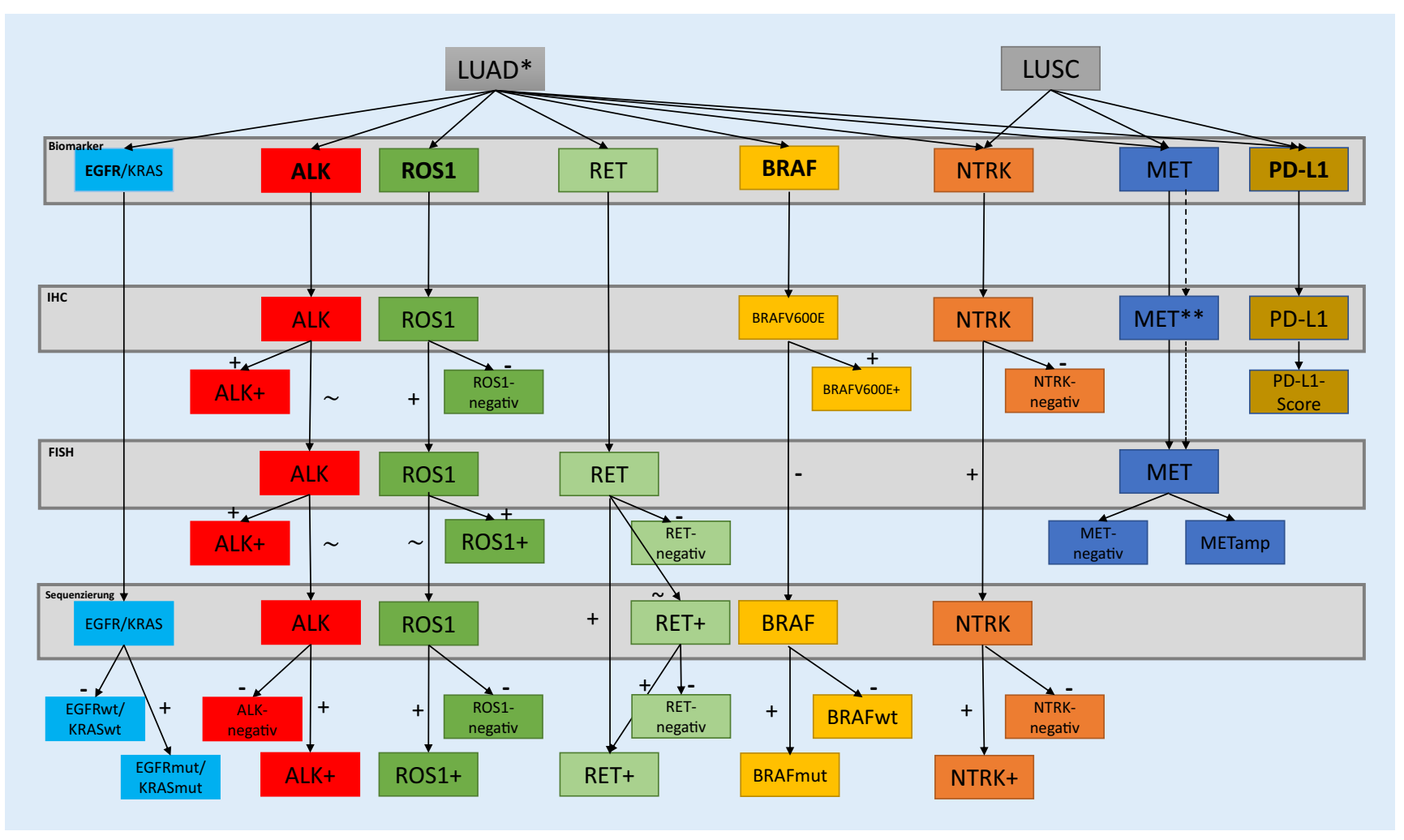

Abb. 1 \ Therapierelevante Biomarker: Darstellung der NSCLC-relevanten (NSCLC, nichtkleinzelliges Bronchialkarzinom) Biomarker und der Etappen der molekularen Aufarbeitung. Unabdingbare Biomarker sind in fett hervorgehoben. RET-FISHund NTRK-IHC werden erst bei Negativität der anderen Marker ergänzt. * pulmonale Adenokarzinome und Nicht-Plattenepithel-Histologie; * MET-IHC-Vorscreening; FISH wird zur Bestätigung benötigt; LUAD pulmonales Adenokarzinom, LUSC Plattenepithelkarzinom, wt wildtyp, mut mutiert, amp amplifiziert, + positiv, - negativ, diskrepant/zweifelhaft

Sonde (wie ZytoLight ${ }^{\circledR}$ SPEC ALK/EML4 TriCheck $^{\mathrm{mm}}$, ZytoVision GmbH, Bremerhaven, Deutschland), die zusätzlich EML4 (blau) markiert, erleichtert den Nachweis einer ALK-EML4-Translokation [8]. Visualisiert wird eine ALK-EML4Fusion durch separate grüne und rote Signale mit jeweils einem kolokalisierten blauen Signal (zusätzliches blaues Signal durch Bruch und EML4-Genumlagerung) (• Abb.2). Eine Nicht-EML4-ALKTranslokation weist ein ALK-Bruchmuster ohne Änderung der EML4-Signale auf [22]. Für robust reproduzierbare Ergebnisse sollten Tumorzellen in mindestens 4 verschiedene Gesichtsfeldern ausgezählt werden, da eine gewisse intratumorale Heterogenität vorliegen kann [26]. Positive Studienergebnisse, die zur Zulassung von ALK-TKI führten, motivierten $\mathrm{zu}$ einem Grenzwert von $15 \%$ [13] bezogen auf mindestens 50 ausgewertete Tumorzellen [26]. In der Mehrheit der Fälle kann der ALK-Status im NSCLC anhand des Cutoff von $15 \%$ zuverlässig bestimmt werden. Grenzfälle mit 10-20\% ALK-positiven Tumorzellen sollten reevaluiert und mittels einer weiteren Testmethode untersucht werden [26]. Diagnostisch anspruchsvoll sind zudem Tumoren mit einem isolierten $3^{\prime}$-Signalmuster beruhend auf einer Deletion des $5^{\prime}$-Signals nach Neuanordnung der $A L K$-Tyrosinkinase-Domäne im $3^{\prime}$-Ende. Diese machen bis zu $5 \%$ der positiven Fälle aus. Die TriCheck ${ }^{\mathrm{m}}$ Sonde kann hier das Vorliegen einer ALK/EML4-Fusion bestätigen [22]. Das extrem selten vorkommende Signalmuster mit isolierten $5^{\prime}$-Signalen wurde jedoch initial als negativ gewertet [26]. In kleinen Fallserien wurden bei Tumoren mit intratumoral $60-87 \%$ isolierten $5^{\prime}$ Signalen und positiver ALK-IHC eine ALK-Translokation mittels sequenzierungsbasierten Verfahren nachgewiesen und die Patienten erfolgreich mit Crizotinib therapiert [6]. Dies unterstreicht die Bedeutung der Validierung schwieriger Signalmuster mittels orthogonaler
Methoden. Hierfür empfiehlt sich vor allem die (RNA-)sequenzierungsbasierte Fusionsdetektion. Bei gängigeren $A L K$ Mustern ist die IHC sequenzierungsbasierten Methoden gleichwertig, da die immunhistochemische ALK-Proteinexpression überzeugend mit dem Vorhandensein einer ALK-Translokation korreliert, sodass die ALK-IHC als Screeninginstrument und sogar als Primärtest für die Auswahl von Patienten für die ALK-TKI-Therapie zugelassen ist $[16,20]$. Über die IHC ist allerdings die Identifikation des jeweiligen Fusionspartners oder der jeweiligen $A L K$ Variante nicht möglich, was offenbar prognostische Relevanz hat [15] und von großem akademischem Interesse ist. Aktuell hat der Nachweis der Variante im klinischen Alltag bei der Primärdiagnostik jedoch keine therapeutische Konsequenz und wird daher nicht routinemäßig durchgeführt.

Alle nicht eindeutigen Fälle (ungewöhnliches Signalmuster oder gängiges/ 
beschriebenes Signalmuster, aber Diskrepanz zwischen ALK-FISH und ALKIHC) sollten immer ergänzend durch sequenzierungsbasierte Methoden getestet werden [16].

\section{ROS1}

Weit über 20 ROS1-Fusionspartner sind bekannt, dabei liegt der größte Teil nicht wie ROS1 auf Chromosom 6. Etwa 1-2\% der pulmonalen Adenokarzinome zeigen eine ROS1-Translokation [26]. Der diagnostische Goldstandard ist die FISH mittels einer Dual-ColourBreak-Apart-FISH-Sonde. Diverse Probensets sind kommerziell erhältlich, die den $5^{\prime}$-Teil (telomer) und den $3^{\prime}$-Teil (zentromer) von ROS1 erkennen, dabei variieren die Genabschnitte, die von den Sonden erfasst werden [26]. In allen bekannten Fusionsgenen ist die ROS1Tyrosinkinase-Domäne am 3'-Ende [9]. Hier existieren 2 positive ROS1-Signalkategorien (•Tab. 1): Das „klassische“ Bruchmuster mit separaten grünen und roten Signalen im Mindestabstand von einem Signaldurchmesser (• Abb. 2) und das „atypische“ Muster mit isolierten 3'Signalen [9]. Der Grenzwert für ein positives Ergebnis liegt beim Nachweis von $15 \%$ und mehr Tumorzellen mit „klassischem“ Bruchmuster oder „atypischem“ Muster [26]. Das atypische Muster, das in einer großen Kohorte von ROS1translozierten LUAD in annähernd 50\% vorkommen kann, birgt die Gefahr eines falsch positiven Ergebnisses [9]. Somit sollten alle atypischen Muster mittels eines orthogonalen Verfahrens validiert werden, wobei hier sequenzierungsbasierte Tests empfehlenswert sind. Die ROS1-IHC eignet sich aufgrund einer hohen Sensitivität für eine ROS1Translokation, bei allerdings geringer Spezifität [16, 20, 26] nur bedingt zur Validierung untypischer Muster. Sie kann aber zum Vorscreening herangezogen werden und insgesamt somit die Zahl der notwendigen FISH-Untersuchungen reduzieren.

Die Bedeutung eines nach konventionellem Grenzwert knapp positiven ROS1-FISH-Ergebnis bei gleichzeitig positiver ROS1-IHC und zusätzlichem Nachweis einer weiteren begleitenden

Wien klin Mag 2021 · 24:34-42 https://doi.org/10.1007/s00740-020-00370-0

(c) Der/die Autor(en) 2020

\section{Hieggelke · A. M. Schultheis}

\section{Anwendungen der FISH in der Diagnostik von Lungenkarzinomen}

\section{Zusammenfassung}

Die rasante Entwicklung im Bereich der Lungenkrebstherapie wurde maßgeblich auch durch die Entdeckung molekularer Marker und der damit verbundenen Möglichkeit einer personalisierten Therapie bestimmt. Die heutige Lungenkrebsdiagnostik stellt hohe Anforderungen an den Pathologen. An kleinen Gewebeproben muss nicht nur die Diagnose gestellt, sondern müssen auch alle therapierelevanten Biomarker getestet werden. Das verlangte Mindestmaß bei fortgeschrittenem nichtkleinzelligen Lungenkarzinom („non small cell lung cancer", NSCLC) mit Nicht-PlattenepithelHistologie umfasst die Testung von EGFR, $B R A F, A L K, R O S 1$ und PD-L1. Für Plattenepithelkarzinome ist bislang nur die PD-L1-IHC (Immunhistochemie, IHC) gefordert. Nach Möglichkeit sollten neuere Biomarker wie RET, MET, HER2, NTRK und KRAS integriert werden. Die Fluoreszenz-in-situ-Hybridisierung („fluorescence in situ hybridization“, FISH) ist eine gut-etablierte Methode zum Nachweis einer $A L K$-, ROS1- und RET-Translokation, wobei die ALK-IHC als gleichwertig anerkannt wurde. Die Relevanz der MET-FISH zum Amplifikationsnachweis im First-line-Setting ist umstritten. Nicht eindeutige Fälle sollten immer mit einem orthogonalen Verfahren validiert werden. Hierzu eignet sich bei $A L K$ und ROS1 die IHC mit dem Vorteil schneller und kostengünstiger Testergebnisse sowie geringen Gewebeverbrauchs. Bei allen anderen Translokationen oder bei Diskrepanz zwischen IHC und FISH sollte ein sequenzierungsbasiertes Verfahren ergänzt werden. Zur Detektion der seltenen NTRK-Fusionen eignet sich bei hoher Sensitivität ein IHCVorscreening; die sequenzierungsbasierte Analyse ist hier bei Positivität zur Bestätigung indiziert.

\section{Schlüsselwörter}

Fluoreszenz-in-situ-Hybridisierung ·

Biomarker - Molekulare Methode .

Bronchialkarzinom · Immunhistochemie

\section{Application of FISH in the diagnosis of lung cancer}

\section{Abstract}

Rapid advancements in the area of lung cancer therapy were determined by the discovery of molecular markers and the possibility of their therapeutic exploitation. Today's lung cancer diagnosis places high demands on pathologists. In the majority of cases, small tissue samples must suffice for diagnosis and testing of all relevant biomarkers. The minimum panel required for advanced non-small-cell lung carcinoma (NSCLC) with nonsquamous histology includes testing of $E G F R, B R A F, A L K, R O S 1$, and PD-L1-expression. So far, only PD-L1$\mathrm{IHC}$ (immunohistochemistry, IHC) is required for squamous cell carcinoma. If possible, newer biomarkers such as RET, MET, HER2, NTRK, and KRAS should be integrated in test panels. Fluorescence in situ hybridization (FISH) is a well-established molecular method for the detection of chromosomal aberrations, such as ALK-, ROS1-, and RETtranslocations and amplifications, such as
Her2/neu or MET. The relevance of METFISH for the detection of amplifications in the first-line setting is controversial, but of high importance in the recurrent setting. All equivocal or discrepant results should be validated using orthogonal methods. IHC is a suitable, thoroughly validated method for $A L K$ and ROS1 aberration detection with the advantage of quick and cost-efficient test results and tissue conservation. All other translocations, or discrepancy between $\mathrm{IHC}$ and $\mathrm{FISH}$, require a sequencing-based confirmation procedure. The low frequency of NTRK fusions, and high sensitivity of NTRK$\mathrm{IHC}$, suggest using $\mathrm{IHC}$ as a prescreening tool with subsequent sequencing-based analysis for IHC positive cases.

\section{Keywords}

Fluorescence-in-situ hybridization - Biomarker - Molecular methods · Lung cancer . Immunohistochemistry 
Hier steht eine Anzeige.

\section{曾 Springer}


Tab. 1 Fluoreszenz-in-situ-Hybridisierungs(FISH)-Signalmuster: Schematische Übersicht der Signalmuster der ALK-/ROS1-und RET-FISH-Sonde und ihre Interpretation bezügliche des Vorliegens einer Translokation

\begin{tabular}{|c|c|c|c|c|}
\hline \multicolumn{2}{|c|}{ Sonden/Norm-Signalverteilung } & \multicolumn{2}{|c|}{ Aberrante Signalverteilung } & \multirow{2}{*}{$\begin{array}{l}\text { Interpretation } \\
\text { ALK-Translokationsstatus positiv ( } \geq 15 \% \text { Bruchsignale in den } \\
\text { Tumorzellen) }\end{array}$} \\
\hline$A L K$ & $A L K-E M L 4$ & $\begin{array}{l}\text { Nicht-ALK-EML4- } \\
\text { Translokation }\end{array}$ & $\begin{array}{l}\text { ALK-EML4- } \\
\text { Inversion }\end{array}$ & \\
\hline & & \multicolumn{2}{|c|}{ ALK isolierte $3^{\prime}$-Signale (rot) } & $A L K$-Translokationsstatus ( $\geq 15 \% A L K$-isolierte $3^{\prime}$-Signale) \\
\hline & & Ohne EML4 & Mit EML4 & Bestätigung durch sequenzierungsbasiertes Verfahren \\
\hline & & $A L K$ isolierte $5^{\prime}-\mathrm{Si}$ & nale (grün) & $\begin{array}{l}\text { ALK-Translokationsstatus ( } \geq 15 \% A L K \text {-isolierte } 5^{\prime} \text {-Signale) in } \\
\text { Kombination mit einer positiven ALK-Immunhistochemie ein } \\
\text { sequenzierungsbasiertes Verfahren anschließen }\end{array}$ \\
\hline \multirow[t]{3}{*}{ ROS1 und RET } & & ROSI1-/RET-Trans & kation & $\begin{array}{l}\text { ROS1-Translokationsstatus positiv ( } \geq 15 \% \text { Bruchsignale in den } \\
\text { Tumorzellen); } \\
\text { RET-Translokationsstatus ( } \geq 20 \% \text { Bruchsignale in den Tumorzellen) }\end{array}$ \\
\hline & & ROSI1-/RET-isolie & e $3^{\prime}$-Signale (grün) & $\begin{array}{l}\text { ROS1-Translokationsstatus positiv (ROS1-isolierte } 3^{\prime} \text {-Signale } \geq 20 \% \text { ); } \\
\text { RET-Translokationsstatus (RET-isolierte } 3^{\prime} \text {-Signale } \geq 20 \% \text { ) } \\
\text { Bestätigung mittels sequenzierungsbasierten Verfahrens }\end{array}$ \\
\hline & & $\begin{array}{l}\text { ROS1-/RET- atypi } \\
\text { must (isolierte } 5^{\prime}\end{array}$ & $\begin{array}{l}\text { he Signal- } \\
\text { ignale (rot)) }\end{array}$ & $\begin{array}{l}\text { ROS1-/RET-Translokationsstatus zweifelhaft; bei allen konstant } \\
\text { aberranten Mustern u. a. isolierte } 5 \text { ' Signale in }>20 \% \text { der Zellen sollte } \\
\text { eine Validierung mittels eines orthogonalen/ } \\
\text { sequenzierungsbasierten Verfahrens durchgeführt werden }\end{array}$ \\
\hline
\end{tabular}

Treibermutationen (wie z.B. EGFR) ist nicht abschließend geklärt [29].

\section{RET}

Die Prävalenz der RET-Translokation beträgt in LUAD 1-2\% [11]. Bislang sollte die Testung nur im Rahmen eines erweiterten Panels oder bei initial negativem EGFR-, ALK- und ROS1-Ergebnis durchgeführt werden [16]. Verschiedene Fusionspartner wurden identifiziert, wobei die RET-Fusion mit KIF5B auf dem gleichen Chromosom (chr10) am besten charakterisiert ist. Analog zur $A L K$ EML4-Fusion liegt eine parazentrische Inversion vor [11]. Allen Fusionen ist die ligandenunabhängige Aktivierung der RET-Tyrosinkinase gemeinsam, was das Ansprechen auf Multikinaseinhibitoren erklärt [11]. Diagnostisch ist die RET-FISH-Analyse eine hochsensitive Nachweismethode einer RET-Fusion [27]. Das häufigste Signalmuster ist ein Fusionssignal neben einem separaten $3^{\prime}$ - und $5^{\prime}$-Signal ([14]; • Abb. 2). In Analogie zu ROS1 sind auch translozierte RET-Fälle mit isoliertem $3^{\prime}$-Signal als prädominiertes FISH-Signalmuster beschrieben, die in einem Kollektiv knapp ein Drittel ausmachen können [14].

Für die RET-IHC besteht bisher keine Anwendungsempfehlung [16], sodass in zweifelhaften Fällen hier immer eine sequenzierungsbasierte Analyse zur Bestätigung einer RET-Fusion angestrebt werden sollte.

Nach Studien ist ein Grenzwert ab $20 \%$ Zellen mit einem aberranten Signalmuster für ein positives Ergebnis plausibel ([14, 27]; - Tab. 1). Die Beurteilung ist besonders herausfordernd, da geteilte RET-Signale zum Teil im Abstand von nur einem Signaldurchmesser liegen [14]. Daher empfiehlt sich besonders bei der RET-FISH die Auswertung durch erfahrene Pathologen/Auswerter.

\section{MET}

MET codiert den „hepatocyte growth factor receptor" (HGFR) und liegt auf Chromosom 7. Initial wurden eine $M E T$ Genaberrationen im Rahmen einer erworbenen Resistenz auf EGFR-TKIs nachgewiesen [4]. Die Rolle von METGenveränderungen als onkogene Treiber ist nicht abschließend geklärt. Bisher hat sich der Nachweis von 2 teilweise überlappenden MET-Alterationen in NSCLC hinsichtlich therapeutischer Ansätze als vielversprechend erwiesen: MET-Exon14(METex14)-skipping-Mutationen, die durch ein Überspringen von Exon 14 einen reduzierten Proteinabbau zur Folge haben [12] und die Amplifikation des MET-Gens [3, 10]. Die Nachweismethode der Wahl einer Amplifikation ist die FISH Analyse. Dual-Colour-FISHSonden markieren das MET-Gen und den zentromeren Teil von Chromosom 7 (CEN 7). Bei der Polysomie ist die Kopieanzahl beider Sonden identisch und 
Hier steht eine Anzeige.

\section{曾 Springer}



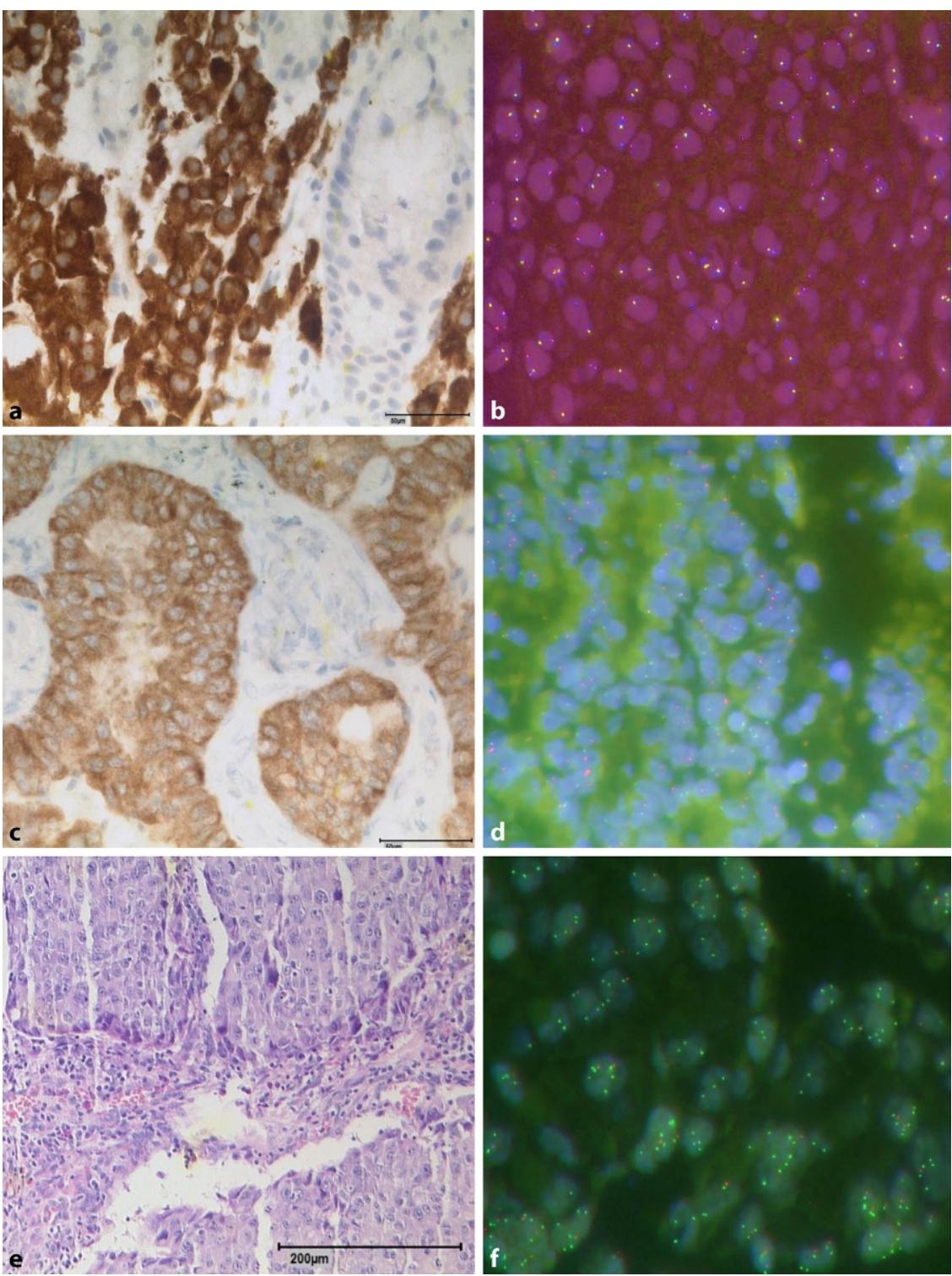

Abb. 2 A Fusionsdetektion. a Kräftig zytoplasmatische Immunhistochemie(IHC)-Positivität für ALK (Klon 1A4, Zytomed Systems GmbH, Berlin, Deutschland) in der Metastase eines pulmonalen Adenokarzinoms (Vergr. 40:1), b korrespondierendes positives ALK-FISH-Ergebnis mit 78\% separaten 3'-Signalen (rot), kolokalisiert mit EML4 (blau, Vergr. 63:1). c Zytoplasmatisch kräftiger ROS1-IHC-Nachweis (Klon D4D6, Cell signaling, Danvers, USA) (Vergr. 40:1), d korrespondierend positive ROS1-FISH (ZytoLight@ SPEC ROS1 Dual Color Break Apart Probe, ZytoVision GmbH, Bremerhaven, Deutschland) mit separaten roten und grünen Signalen (Bruchmuster, Vergr. 63:1). e Gering differenziertes pulmonales Adenokarzinom in der Hämatoxylin-Eosin-Färbung (Vergr. 40:1). f Positive RET-FISH (ZytoLight ${ }^{\circledR}$ SPEC RET Dual Color Break Apart Probe, ZytoVision GmbH, Bremerhaven, Deutschland) mit separaten (extra) grünen Signalen in allen Tumorzellen (Vergr. 63:1) validiert durch einen RET-Translokationsnachweis mittels sequenzierungsbasierter Analyse $\left(\mathrm{ARCHER}^{\circledR}\right.$, Archer FusionPlex, Archer Dx, Boulder, CO, USA)

gesteigert ( $>2 /$ Zelle). Eine echte Amplifikation liegt bei isoliert vermehrten $M E T$ Signalen vor, was die Ratio (MET/CEN 7) anhebt ([10]; • Abb. 3). MET-Amplifikationen sind in gleicher Frequenz in LUAD und LUSC nachzuweisen [21]. Aufgrund von fokal amplifizierten Tumorklonen („hot spots“) und Fällen mit isoliert liert [3, 21]: Neben einem MET/CEN 7Verhältnis $\geq 2,0$ werden Fälle mit einer durchschnittlichen MET-Genkopiezahl von $\geq 5$ bzw. $\geq 6$ als positiv gewertet sowie eine intermediär ( $\geq 50 \%$ mit $\geq 5 \mathrm{MET}$ Genkopien) und eine niedrige $M E T$ Amplifikation ( $\geq 40 \%$ mit $\geq 4$ Kopien) abgegrenzt.

Der Stellenwert der MET-Amplifikation im primären Bronchialkarzinom bleibt jedoch umstritten und nach aktuellen Studien als Prognosefaktor für das Ansprechen einer MET-InhibitorTherapie dem Nachweis einer METex14skipping-Mutation unterlegen [30]. Die therapeutische Rolle einer Top-LevelMET-Amplifikation (durchschnittlich $\geq 10 \mathrm{MET}$ ) in LUSC, die mit einer besonders schlechten Prognose assoziiert ist [18], bleibt noch offen. Nach aktueller Empfehlung soll die MET-Analyse integriert in erweiterte Biomarkerpanels nach negativem Ergebnis für $E G F R, A L K$ und ROS1 und vor allem in der Resistenzsituation (s. Abschn. „Diagnostik bei erworbener Arzneimittelresistenz") durchgeführt werden [16]. Die METIHC ist nicht standardisiert und korreliert nur mit einer $M E T$-Amplifikation, während das IHC-Ergebnis bei einer MET-Exon-14-skipping-Mutation heterogen und offenbar ohne Vorhersagewert ist [2].

\section{Neue Biomarker}

$\mathrm{Zu}$ den neueren Biomarkern gehören vor allem die Gene der neurotrophen Tropomyosinrezeptorkinase (NTRK) NTRK1, NTRK2 und NTRK3 -, die 3 TRK-Proteine (TRKA, TRKB und TRKC) codieren. NTRK-Translokationen treten in bis zu $4 \%$ der NSCLC auf [28]. Vielversprechende Studienergebnisse führten bereits zur FDA-Zulassung von Larotrectinib, einem selektiven panTRK-Inhibitor [28]. Der Nachweis im Rahmen der Zulassungsstudien erfolgte NGS-basiert oder mittels FISH. Eine Multiplex-FISH mit 3 separaten FISHAssays ist technisch möglich und aussagekräftig, aber teuer, zeitaufwendig und erfordert viel Erfahrung bei der Interpretation. Gut in der Diagnostik etabliert ist bisher nur die ETV6-NTRKFISH [17]. Die panNTRK-IHC zeigte 

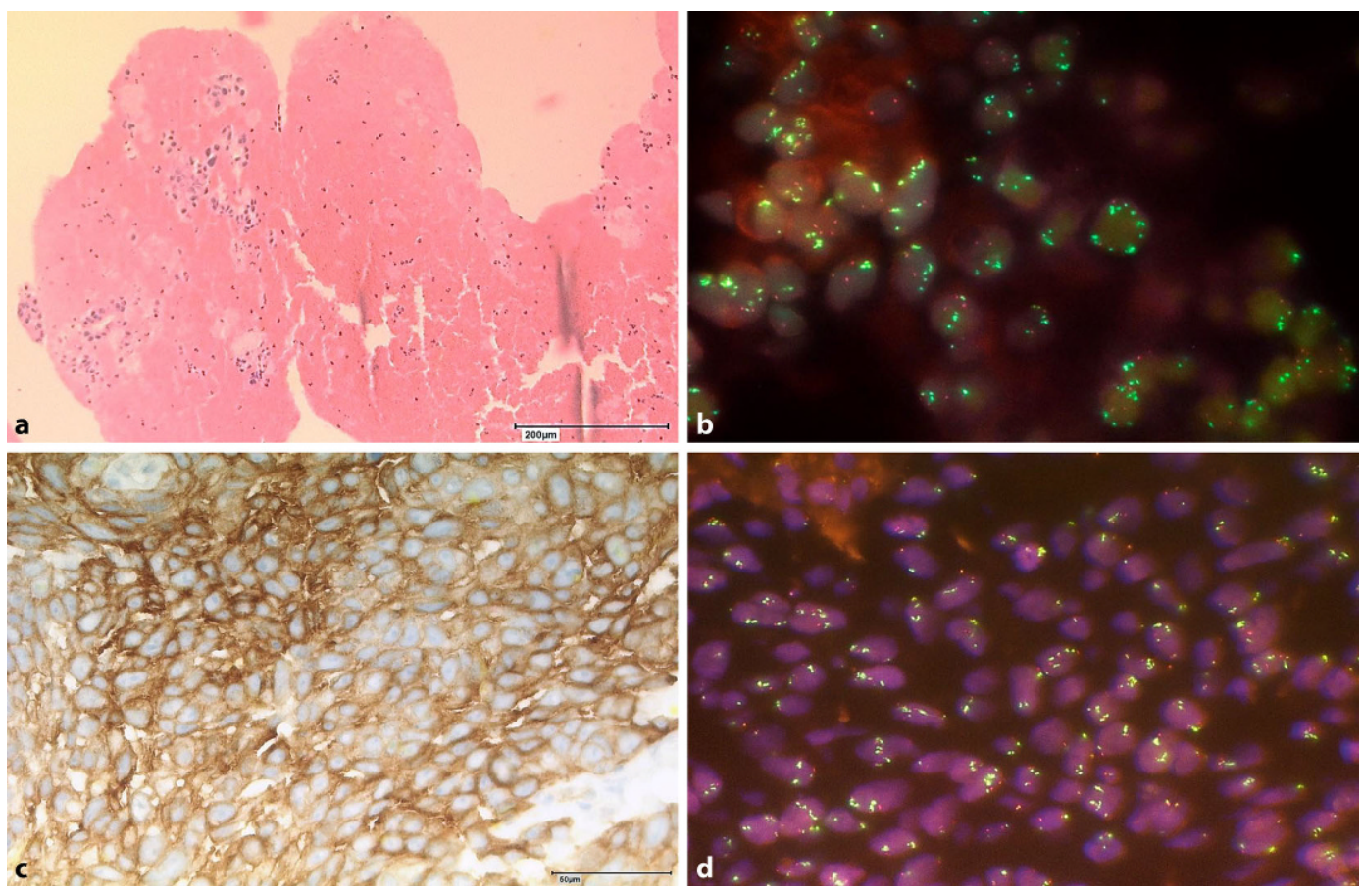

Abb. $3 \triangleleft$ MET-Nachweis. a Feinnadelpunktion eines pulmonalen Adenokarzinoms (HE-Färbung, Vergr. 40:1). b Nachweis der MET-Amplifikation mit Ratio (MET/CEN 7) 2,06 und durchschnittlicher MET-Genkopiezahl von 12,15 mittels FISH (ZytoLight ${ }^{\circledR}$ SPEC MET/CEN 7 Dual Color Probe, ZytoVision $\mathrm{GmbH}$, Bremerhaven, Deutschland) (Vergr. 63:1) c Kräftige zytoplasmatische MET-IHC-Positivität (Klon SP44, Roche, Basel, Schweiz) (Vergr. 40:1). d Korrespondierende MET-Amplifikation mit Ratio (MET/CEN 7) von 3,7 und durchschnittlicher MET-Genkopiezahl von 7,70 (Vergr. 63:1)

in Studien einen zuverlässigen Nachweis aller NTRK-Fusionen und wird daher vor allem zum Vorscreening einer NTRK-Fusion empfohlen [7], wobei schon der geringste IHC-Proteinnachweis eine Multipanel-NTRK-Genanalyse oder FISH-Analyse nach sich ziehen sollte [17].

\section{Diagnostik bei erworbener Arzneimittelresistenz}

Die erworbene Arzneimittelresistenz bleibt ein kritisches Hindernis für die Wirksamkeitsmaximierung gezielter Therapien im NSCLC. $\mathrm{Zu}$ den Mechanismen gehören zielabhängige und zielunabhängige Veränderungen. Der Minimalalgorithmus für die TKI-Resistenztestung ist nicht nur von akademischem Interesse, sondern maßgeblich für klinische Therapieentscheidungen und umfasst den Nachweis/die Bestätigung des initialen Treibermechanismus (EGFR-Mutation, ALK- oder ROS1-Translokation) sowie zusätzlich die Detektion einer möglichen $M E T$-, HER2- oder ALK-Amplifikation als Resistenzmechanismus. Analog zur METFISH markieren Dual-Colour-Sonden das Zielgen (ALK/HER2) und das zugehörige Zentromer (CEN 2/CEN 17). Der Grenzwert für eine HER2-Amplifikation liegt bei einer Ratio (HER2/CEN 17) von $\geq 2,2$ oder einer Genkopiezahl von $>15$ in $10 \%$ der Zellen. Fälle im Graubereich (Ratio 1,8-2,2) sollten eingehend kontrolliert und mittels eines weiteren Verfahrens eingeordnet werden [25]. Eine ALK-Amplifikation wird bereits ab einer Ratio $>2$ angenommen [5].

Die Detektion von (High-level-)METAmplifikationen unter TKI- Resistenzentwicklung hat einen besonders hohen Stellenwert, da diese therapeutisch adressierbar sind (Kombination aus EGFRund MET-TKI), und ist möglicherweise sogar höher einzuschätzen als in der Primärdiagnostik.

\section{Fazit für die Praxis}

- Fluoreszenz-in-situ-Hybridisierung (FISH) ist eine präzise, schnelle und relativ kostengünstige Nachweismethode von relevanten Genveränderungen im nichtkleinzelligen Lungenkarzinom (NSCLC).

- Bei der ALK-Testung darf die ALK-IHC (Immunhistochemie) als gleichwertig angesehen werden, eine Bestätigung mittels FISH-Analyse ist allerdings trotzdem angeraten.

- Für andere Biomarker (ROS1, RET) korreliert die IHC nicht eindeutig mit der Translokation und FISH bleibt die Methode der Wahl.

- Next-Generation-Sequencing(NGS)basierte Sequenzierungsverfahren sollten bei allen diskrepanten/ ungewöhnlichen FISH-Fällen hinzugezogen werden.

- Beim Nachweis von Amplifikationen (u. a. MET) ist die FISH den sequenzierungsbasierten Verfahren bisher überlegen.

- Für alle FISH Analysen ist die gute Vorkenntnis des Untersuchers essenziell; die Signalmuster der Sonden müssen genau gekannt werden, die Ergebnisse im Gesamtkontext der Erkrankung interpretiert und hinterfragt werden.

- Die Erstellung eines FISH-Befundes mit aussagekräftigem Ergebnis, aber auch unter Angabe des verwendeten Sondensystems, der Anzahl der gezählten Tumorzellen, des Schwellenwertes und auch ggf. Besonderheiten/Schwierigkeiten ist wünschenswert.

\section{Korrespondenzadresse}

Prof. Dr. Anne M. Schultheis

Institut für Pathologie, Uniklinik Köln Kerpener Str. 62, 50937 Köln, Deutschland anne.schultheis@uk-koeln.de 
Funding. Open Access funding enabled and organized by Projekt DEAL.

\section{Einhaltung ethischer Richtlinien}

Interessenkonflikt. L. Hieggelke und A.M. Schultheis geben an, dass kein Interessenkonflikt besteht.

Für diesen Beitrag wurden von den Autoren keine Studien an Menschen oder Tieren durchgeführt. Für die aufgeführten Studien gelten die jeweils dort angegebenen ethischen Richtlinien.

Open Access Dieser Artikel wird unter der Creative Commons Namensnennung 4.0 International Lizenz veröffentlicht, welche die Nutzung, Vervielfältigung Bearbeitung, Verbreitung und Wiedergabe in jeglichem Medium und Format erlaubt, sofern Sie den/die ursprünglichen Autor(en) und die Quelle ordnungsgemäß nennen, einen Link zur Creative Commons Lizenz beifügen und angeben, ob Änderungen vorgenommen wurden.

Die in diesem Artikel enthaltenen Bilder und sonstiges Drittmaterial unterliegen ebenfalls der genannten Creative Commons Lizenz, sofern sich aus der Abbildungslegende nichts anderes ergibt. Sofern das betreffende Material nicht unter der genannten Creative Commons Lizenz steht und die betreffende Handlung nicht nach gesetzlichen Vorschriften erlaubt ist, ist für die oben aufgeführten Weiterverwendungen des Materials die Einwilligung des jeweiligen Rechteinhabers einzuholen.

Weitere Details zur Lizenz entnehmen Sie bitte der Lizenzinformation auf http://creativecommons.org/ licenses/by/4.0/deed.de.

\section{Literatur}

1. Agency EM (2012) Eur Med Agency. https://www. ema.europa.eu/en/documents/assessmentreport/xalkori-epar-public-assessment-report_ en.pdf.Zugegriffen: 18. April 2020

2. Castiglione R, Alidousty C, Holz B et al (2019) Comparison of the genomic background of MET-altered carcinomas of the lung: biological differences and analogies. Mod Pathol 32:627-638

3. Drilon A, Cappuzzo F, Ou S-Hl et al (2017) Targeting MET in lung cancer: Will expectations finally be MET? J Thorac Oncol 12:15-26

4. Engelman JA,ZejnullahuK, MitsudomiTetal (2007) MET amplification leads to gefitinib resistance in lung cancer by activating ERBB3 signaling. Science 316:1039-1043

5. Gainor JF, Dardaei L, Yoda S et al (2016) Molecular mechanisms of resistance to first- and secondgeneration ALK inhibitors in ALK-rearranged lung cancer. Cancer Discov 6:1118-1133

6. Guyard A, Charpy C, Théou-Anton N et al (2019) Isolated $5^{\prime}$ signals are an atypical pattern to be considered as positive for ALK rearrangement: a brief report of three cases and review of the literature. TransI Oncol 12:784-787

7. Hechtman JF, Benayed R, Hyman DM et al (2017) Pan-Trkimmunohistochemistry is an efficient and reliable screen for the detection of NTRK fusions. Am J Surg Pathol 41:1547-1551

8. Heydt C, Kostenko A, Merkelbach-Bruse $S$ et al (2016) ALK evaluation in the world of multiplex testing: Network Genomic Medicine (NGM): the Cologne model for implementing personalised oncology. Ann Oncol. Suppl 3:iii25-iii34. https://doi. org/10.1093/annonc/mdw303.PMID:27573753

9. Heydt C, Ruesseler V, Pappesch R et al (2019) Comparison of in situ and extraction-based methods for the detection of ROS1 rearrangements in solid tumors. J Mol Diagnostics 21:971-984

10. Kawakami H, Okamoto I, Okamoto W et al (2014) Targeting MET amplification as a new oncogenic driver. Cancers 6:1540-1552

11. Kohno T, Tsuta K, Tsuchihara K et al (2013) RET fusion gene: translation to personalized lung cancer therapy. Cancer Sci 104:1396-1400

12. Kong-Beltran M, Seshagiri S, Zha J et al (2006) Somatic mutations lead to an oncogenic deletion of met in lung cancer. Cancer Res 66:283-289

13. Kwak EL, Bang YJ, Camidge DR et al (2010) Anaplastic lymphoma kinase inhibition in nonsmall-cell lung cancer. NEnglJMed 363:1693-1703

14. Lee SE, Lee B, Hong M et al (2015) Comprehensive analysis of RET and ROS1 rearrangement in lung adenocarcinoma. Mod Pathol 28:468-479

15. Lin JJ, Zhu VW, Yoda S et al (2018) Impact of EML4ALK variant on resistance mechanisms and clinica outcomes in ALK-positive lung cancer. J Clin Oncol 36:1199-1206

16. Lindeman NI, Cagle PT, Aisner DL et al (2018) Updated molecular testing guideline for the selection of lung cancer patients for treatment with targeted Tyrosine kinase inhibitors: guideline from the College of American Pathologists, the International Association for the Study of Lung Cancer, and the Association for Molecular Pathology. Arch Pathol Lab Med 142:321-346

17. Marchiò C, Scaltriti M, Ladanyi M et al (2019) ESMO recommendations on the standard methods to detect NTRK fusions in daily practice and clinical research. Ann Oncol 30(9):1417-1427. https://doi. org/10.1093/annonc/mdz204.PMID:31268127

18. OverbeckTR,CronDA,SchmitzKetal (2020) Top-level MET gene copy number gain defines a subtype of poorly differentiated pulmonary adenocarcinomas with poor prognosis. Transl Lung Cancer Res 9(3):603-616. https://doi.org/10.21037/tlcr-19339.PMID:32676323;PMCID:PMC7354108

19. Paez JG, Janne PA, Lee JC et al (2004) EGFR mutations in lung cancer: correlation with clinical response to gefitinib therapy. Science 304:1497-1500

20. Planchard D, Popat S, Kerr Ket al (2018) Metastatic non-small cell lung cancer: ESMO Clinical Practice
Guidelines for diagnosis, treatment and follow-up. Ann Oncol 29:iv192-iv237

21. Schildhaus $\mathrm{H}-U$, Schultheis $A M$, Rüschoff J et a (2015) MET amplification status in therapy-Naïve Adeno- and Squamous cell carcinomas of the lung. Clin Cancer Res 21:907-915

22. Smuk G, Pajor G, Szuhai K et al (2020) Attenuated isolated $3^{\prime}$ signal: a highly challenging therapy relevant ALK FISH pattern in NSCLC. Cancer Treat Res 143:80-85

23. Society AC (2020) Key statistics for lung cancer/ What is lung cancer? https://www.cancer.org/ cancer/lung-cancer/about/key-statistics.html. Zugegriffen: 18. April 2020

24. Soda M, Choi YL, Enomoto M et al (2007) Identification of the transforming EML4-ALK fusion gene in non-small-cell lung cancer. Nature 448:561-566

25. Takezawa K, Pirazzoli V, Arcila ME et al (2012) HER2 amplification: a potential mechanism of acquired resistance to EGFR inhibition in EGFR-mutant lung cancers that lack the second-site EGFR T790M mutation. Cancer Discov 2:922-933

26. Tsao MS, Hirsch FR, Yatabe Yetal (2016) IASLC Atlas of ALK and ROS1 testing in lung cancer. Editorial Rx Press, Aurora, Colorado

27. Tsuta K, Kohno T, Yoshida A et al (2014) RET-rearranged non-small-cell lung carcinoma: a clinicopathological and molecular analysis. $\mathrm{Br}$ Cancer 110:1571-1578

28. Wang W, Xu C, Zhu Y et al (2018) P2.03-09 the rea world of NTRK fusion data in the Chinese lung cancer populations: a multicenter study. J Thorac Oncol 13:S719

29. Wiesweg M, Eberhardt WEE, Reis Hetal (2017) High prevalence of concomitant oncogene mutations in prospectively identified patients with ROS1positive metastatic lung cancer. J Thorac Onco 12:54-64

30. Wolf J, Seto T, Han J-Y et al (2019) Capmatinib (INC280) in MET $\Delta$ ex14-mutated advanced nonsmall cell lung cancer (NSCLC): Efficacy data from the phase II GEOMETRY mono-1 study. J Clin Oncol 37:9004-9004

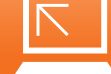

Strategische Partnerschaft

Niedermolekulare Wirkstoffe sollen durch die Nutzung einer auf generativer Chemie basierenden Plattformtechnologie schneller entdeckt werden. Für diese Strategie haben das auf maschinelle Lernlösungen für die präklinische Arzneimittelforschung spezialisierte Biotechnologieunternehmen PostEra und das Pharmaunternehmen Pfizer eine mehrjährige strategische Partnerschaft geschlossen. Hochmoderne, skalierbare Modelle, die die umfangreichen Daten und das Fachwissen sowie die technologischen Möglichkeiten der beiden Partner verknüpfen, sollen in der präklinischen Wirkstoffforschung eingesetzt werden 